International Journal of Pure and Applied Mathematics

Volume 90 No. 2 2014, 165-175

ISSN: 1311-8080 (printed version); ISSN: 1314-3395 (on-line version)

url: http://www.ijpam.eu

doi: http://dx.doi.org/10.12732/ijpam.v90i2.6

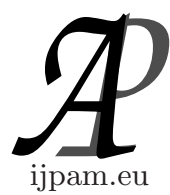

\title{
A NOTE ON THE INTEGRAL REPRESENTATION OF THE VALUE FUNCTION
}

\author{
Marcelo de C. Griebeler ${ }^{1}$, Jorge Paulo de Araújo ${ }^{2}$ \\ ${ }^{1}$ Department of Economics \\ Santa Catarina State University \\ Florianópolis, ZIP Code: 88035-001, BRAZIL \\ ${ }^{2}$ Department of Economics \\ Federal University of Rio Grande do Sul \\ Porto Alegre, ZIP Code: 90040-000, BRAZIL
}

\begin{abstract}
In this paper we propose an alternative assumption for the integral representation of the value function of Milgrom and Segal (2002). Instead of requiring that utility function has derivative almost everywhere, we impose that it has derivative in all its domain. The idea is to obtain conditions in order to apply the Lebesgue Theorem which provides at the same time an absolutely continuous value function and its integral representation. Our assumption is technically stronger than that of Milgrom and Segal (2002) but we argue that there is a substantial gain of economic interpretation in adding it. While it is difficult to interpret absolute continuity in terms of agent's preferences, the existence of the derivative everywhere means that all agent's choices are smooth.
\end{abstract}

AMS Subject Classification: 28A10, 91A80, 91A25

Key Words: value function, mechanism design, differentiability

\section{Introduction}

Representing the value function in its integral form is a fundamental step in

Received: September 18, 2013

(c) 2014 Academic Publications, Ltd.

${ }^{\S}$ Correspondence author url: www.acadpubl.eu 
the process of solving mechanism design models with continuous type spaces. This step is part of an alternative approach first suggested by [9] in his classic study about optimal taxation. Instead of using optimal control, in that paper is proposed a three-steps process of solution. The idea is to eliminate one variable in the principal's optimization problem by using the indirect utility, provided by the value function. This is done by applying the Envelope Theorem (first step) and by writing the indirect utility in an integral form, in the second step. Thus, in the third step it is possible to replace this integral in the objective function and obtain a simplified expression to optimize.

Since the seminal paper of [9] this approach has been used in studies applied to all fields of Economics. Examples are [10] in price discrimination, [2] in Economics of Regulation and [7] in non-linear monopoly precification. More recently, [14] has provided some theoretical advances using that framework. All these papers have in commom the standard assumption of differentiability of mechanisms. In fact, this requirement is crucial in the first step of the approach because the Envelope Theorem does not hold without differentiability. However, several examples in many differents fields of Economics have showed that assumption may not be satisfactory. [6] and [11], for example, present discontinuous optimal mechanisms in Regulation Economics and bilateral trading, respectively.

Literature has tried to obtain the Envelope Theorem and the integral representation of the value function without differentiability. In this context, important studies are [5], [3] and [4]. They focus their attention in developing general forms of the "payoff equivalence", with a particular case being the Revenue Equivalence Theorem. That literature has the advantage of allowing applications to multidimensional type spaces. However, by restricting the analysis to "payoff equivalence" its findings apply only to settings where the agent's utility is separable in the payment (or transfer) function. In other words, in order to obtain an integral representation of the value function, these authors require that the preferences are quasi-linear.

[8] are more general than the remainder of the literature because they obtain the two first steps of the approach of [9] without differentiability and allowing that the utilities assume any functional form. In the other hand, the disadvantage of their study is the fact that it only applies to mechanism design models with a single agent. This limitation is overcame by [1], that expand their Envelope Theorem to multidimensional type spaces by imposing an additional assumption. Through that new requirement those authors obtain a direct generalization of the integral representation of the value function. Nevertheless, according to [3], the assumption of absolute continuity assumed by these two 
papers to write the value function as an integral is not elegant. In addition, it has no economic interpretation.

In this paper we propose an alternative assumption for integral representation of the value function of [8]. Instead of requiring that utility function has derivative almost everywhere, we impose that it has derivative in all its domain. The idea is to obtain conditions in order to apply the Lebesgue Theorem which provides at the same time an absolute continuous value function and its integral representation. Our assumption is technically stronger than that of [8] but we argue that there is a substantial gain of economic interpretation in adding it. While it is difficult to interpret absolute continuity in terms of agent's preferences, the existence of the derivative everywhere means that all agent's choices are smooth.

Our paper is divided in two sections besides this brief introduction. In the second section we require the existence of the derivative everywhere in the agent's utility and obtain the same integral representation of the rest of literature. In order to do so we use a modified version of the Vitali-Carathéodory Theorem and the Lebesgue Theorem. We also argue that our assumption has more economic interpretation than that of [8]. Section three concludes.

\section{Main Results}

As the literature cited in introduction shows, existence almost everywhere of the utility function's derivative is a consequence of the assumption of absolute continuity. Here we make a technically stronger requirement, namely we require that agent's utility function has derivative everywhere. There are two reason to include it. The first one is that with this additional assumption we have more economic intepretation. Instead of existing almost everywhere, the agent's marginal utility with respect to his type exists everywhere. It is very plausible that for any type chosen by the agent his utility varies locally.

The other reason is that we are only worried in allowing the mechanism be arbitrary, following the related literature. Thus, in that sense, it is not a limitation impose some structure in the preferences. In fact, in applications it is difficult to see an example of utility functions that violate the existence of the derivative everywhere. Therefore we do so below. However, before that we need some auxiliaries results ${ }^{1}$.

Lemma 1. Let $f: X \rightarrow[0,+\infty]$ be a mensurable function, then there exists a sequence $\left(\varphi_{n}\right)$ of simple functions such that $\varphi_{n}(x) \uparrow f(x)$.

\footnotetext{
${ }^{1}$ Mathematical concepts used in this section may be found in [12] and [13].
} 
Proof. Let's start by defining the following sets:

$$
\begin{aligned}
E_{1}= & \{x \in X \mid f(x) \geq 1\} \\
E_{2}= & \left\{x \in X \mid f(x) \geq \frac{1}{2}+\chi_{E_{1}}(x)\right\} \\
& \vdots \\
E_{k}= & \left\{x \in X \mid f(x) \geq \frac{1}{k}+\sum_{j=1}^{k-1} \chi_{E_{j}}(x)\right\},
\end{aligned}
$$

for $k \geq 2$ and where $\chi_{E_{j}}(\cdot)$ is the characteristic function of the set $E_{j}$. Notice that since $E_{1}$ is mensurable so is $E_{2}$, because we may write $f(x)-\chi_{E_{1}}(x) \geq 1$. Doing the same reasoning we obtain that $E_{k}$ is mensurable for every $k$.

Now we define the function $\varphi_{k}(x)=\sum_{j=1}^{k} \frac{1}{j} \chi_{E_{j}}(x)$. Clearly, $\varphi_{k}(\cdot)$ is monotonically nonincreasing because $\varphi_{k}(x)=\sum_{j=1}^{k} \frac{1}{j} \chi_{E_{j}}(x) \leq \varphi_{k+1}(x)=\sum_{j=1}^{k+1} \frac{1}{j} \chi_{E_{j}}(x)$. We must demonstrate that $\varphi_{k}(x) \leq f(x)$.

Suppose by absurd that $\varphi_{k}(x)>f(x)$. Thus, there exist $k_{0}$ and $x^{\prime}$ such that $0 \leq f\left(x^{\prime}\right)<\varphi_{k_{0}}\left(x^{\prime}\right)$, implying $\varphi_{k_{0}}\left(x^{\prime}\right)=\sum_{j=1}^{k_{0}} \frac{1}{j} \chi_{E_{j}}\left(x^{\prime}\right)>0$. Then, $x^{\prime} \in E_{j}$ for some $1 \leq j \leq k_{0}$. Set $k^{\prime}=\max _{x^{\prime} \in E_{j}} j$. If $k^{\prime}=1$ so $f\left(x^{\prime}\right) \geq 1=\varphi_{E_{1}}\left(x^{\prime}\right)$. Similarly, if $k \geq 2$ we have $x^{\prime} \in E_{k^{\prime}}$, such that

$$
f\left(x^{\prime}\right) \geq \frac{1}{k^{\prime}}+\sum_{j=1}^{k^{\prime}-1} \frac{1}{j} \chi_{E_{j}}\left(x^{\prime}\right)=\sum_{j=1}^{k^{\prime}} \frac{1}{j} \chi_{E_{j}}\left(x^{\prime}\right)=\varphi_{k^{\prime}}\left(x^{\prime}\right) .
$$

Therefore we have $f\left(x^{\prime}\right) \geq \varphi_{k^{\prime}}\left(x^{\prime}\right)$ e $k^{\prime}<k_{0}$, because by assumption $f\left(x^{\prime}\right)<$ $\varphi_{k_{0}}\left(x^{\prime}\right)$. Using the definitions,

$$
\begin{aligned}
0 & \leq f\left(x^{\prime}\right)-\varphi_{k^{\prime}}\left(x^{\prime}\right)=f\left(x^{\prime}\right)-\sum_{j=1}^{k^{\prime}} \frac{1}{j} \chi_{E_{j}}\left(x^{\prime}\right) \\
& <\sum_{j=1}^{k_{0}} \frac{1}{j} \chi_{E_{j}}\left(x^{\prime}\right)-\sum_{j=1}^{k^{\prime}} \frac{1}{j} \chi_{E_{j}}\left(x^{\prime}\right) \\
& =\sum_{j=k^{\prime}+1}^{k_{0}} \frac{1}{j} \chi_{E_{j}}\left(x^{\prime}\right) .
\end{aligned}
$$


However, the fact that $0<\sum_{j=k^{\prime}+1}^{k_{0}} \frac{1}{j} \chi_{E_{j}}\left(x^{\prime}\right)$ implies that there exists a $k^{\prime \prime}>$ $k^{\prime}$ such that $x^{\prime} \in E_{k^{\prime \prime}}$, what contradicts our initial assumption that $k^{\prime}$ is the maximum. Thus, $\varphi_{k}(x) \leq f(x)$.

We still need to show that $\varphi_{n}(x) \uparrow f(x)$. In the case of $f(x)=+\infty$, $x \in E_{j}$ for every $j$, such that $\varphi_{n}(x)=1+\frac{1}{2}+\ldots+\frac{1}{n} \rightarrow \infty$ as $n \rightarrow \infty$. When $f(x)<+\infty$, there is no $N$ such that $x \in E_{j}$, for every $j \geq N$, because in the case of existence, $\varphi_{j}(x)=\frac{1}{N}+\frac{1}{N+1}+\ldots+\frac{1}{j} \rightarrow \infty$. Then, there exists $k_{m}$ such that $x \notin E_{j}$ for $j \geq k_{m}$. Therefore,

$$
\begin{aligned}
f(x) & <\frac{1}{k_{m}}+\sum_{j=1}^{k_{m}-1} \frac{1}{j} \chi_{E_{j}}(x) \\
& \Rightarrow 0 \leq f(x)-\varphi_{k_{m-1}}(x)<\frac{1}{k_{m}} \\
& \Rightarrow \varphi_{k_{m-1}}(x) \rightarrow f(x) .
\end{aligned}
$$

Since $\varphi_{n}(x)$ is monotonic, we have $\varphi_{n}(x) \uparrow f(x)$.

The theorem below is a modified version of a result known as Vitali-Carathéodory Theorem.

Theorem 1. Let $f: X \rightarrow[-\infty,+\infty]$ be a mensurable function. Then for all $\varepsilon>0$ there exist $u$ and $v$ such that $u \leq f \leq v$ and

$$
\int_{X}(f-u) d \mu<\varepsilon
$$

and

$$
\int_{X}(v-f) d \mu<\varepsilon,
$$

where $u$ is upper hemicontinuous and $v$ is lower hemicontinuous.

Proof. We first demonstrate in the case of $f(x) \geq 0$. Using Lemma 1, we may write $f(x)=\sum_{j=1}^{\infty} \frac{1}{j} \chi_{E_{j}}(x)$. Since the sets $E_{j}$ are mensurables, for all $\varepsilon>0$, there exists an open $U_{j}$ such that $E_{j} \subseteq U_{j}$ and $\mu\left(U_{j} \backslash E_{j}\right)<\frac{\varepsilon}{2^{j}}$. Thus, we may define

$$
v(x)=\sum_{j=1}^{\infty} \frac{1}{j} \chi_{U_{j}}(x)
$$

and verify that $f(x)=\sum_{j=1}^{\infty} \frac{1}{j} \chi_{E_{j}}(x) \leq \sum_{j=1}^{\infty} \frac{1}{j} \chi_{U_{j}}(x)=v(x)=v$. 
Since that each $U_{j}$ is open then $\frac{1}{j} \chi_{U_{j}}(x)$ and $v(x)$ are lower hemicontinuous, because a series of lower hemicontinuous functions is also lower hemicontinuous.

Now we may obtain (2). Notice that

$$
\begin{aligned}
\int_{X}(v-f) d \mu & =\sum_{j=1}^{\infty} \frac{1}{j} \mu\left(U_{j} \backslash E_{j}\right) \\
& =\sum_{j=1}^{\infty} \frac{1}{j} \frac{\varepsilon}{2^{j}}<\varepsilon .
\end{aligned}
$$

Now let's show (1). For each $E_{j}$ there also exists a closed $K_{j}$ such that $K_{j} \subseteq E_{j}$ and $\mu\left(E_{j} \backslash K_{j}\right)<\frac{\varepsilon}{2^{j}}$. Proceding in the same way above,

$$
\sum_{j=1}^{\infty} \frac{1}{j} \chi_{K_{j}}(x) \leq \sum_{j=1}^{\infty} \frac{1}{j} \chi_{E_{j}}(x)=f(x)
$$

and intregating

$$
\int_{X}\left(f-\sum_{j=1}^{\infty} \frac{1}{j} \chi_{K_{j}}(x)\right) d \mu=\sum_{j=1}^{\infty} \frac{1}{j} \mu\left(E_{j} \backslash K_{j}\right)<\varepsilon .
$$

Notice that it is possible to choose a $N<\infty$ such that

$$
\int_{X}\left(f-\sum_{j=1}^{N} \frac{1}{j} \chi_{K_{j}}(x)\right) d \mu<\varepsilon .
$$

Defining

$$
u(x)=\sum_{j=1}^{N} \frac{1}{j} \chi_{K_{j}}(x),
$$

we have that $u(x)$ is upper hemicontinuous because $K_{j}$ is closed. Thus,

$$
\int_{X}(f-u) d \mu<\varepsilon .
$$

In the general case, with $-\infty \leq f(x) \leq+\infty$, we separate $f$ in its positive and negative parts, $f=f^{+}-f^{-}$. Since both parts are nonnegative we must have $u^{+} \leq f^{+} \leq v^{+}$and $u^{-} \leq f^{-} \leq v^{-}$, where the functions $u$ 's are upper hemicontinuous and the $v$ 's are lower hemicontinuous. By changing the signal 
of the functions related to negative parts, we have $-v^{-} \leq-f^{-} \leq-u^{-}$, where now $-u^{-}$is lower hemicontinous and $-v^{-}$is upper hemicontinous. Then,

$$
\hat{u}=u^{+}-v^{-} \leq f \leq v^{+}-u^{-}=\hat{v} .
$$

Moreover,

$$
\begin{aligned}
\int_{X}(f-\hat{u}) d \mu & =\int_{X}\left(\left(f^{+}-f^{-}\right)-\left(u^{+}-v^{-}\right)\right) d \mu \\
& =\int_{X}\left(f^{+}-u^{+}\right) d \mu+\int_{X}\left(v^{-}-f^{-}\right) d \mu \\
& <2 \varepsilon
\end{aligned}
$$

and

$$
\begin{aligned}
\int_{X}(\hat{v}-f) d \mu & =\int_{X}\left(\left(v^{+}-u^{-}\right)-\left(f^{+}-f^{-}\right)\right) d \mu \\
& =\int_{X}\left(v^{+}-f^{+}\right) d \mu+\int_{X}\left(f^{-}-u^{-}\right) d \mu \\
& <2 \varepsilon .
\end{aligned}
$$

Now we enunciate and demonstrate the result that garantees the integral representation of $f$ using the existence of the derivative everywhere.

Theorem 2. Let $f:[a, b] \rightarrow \mathbb{R}$ be a function with derivative in all its domain and let $f^{\prime} \in L^{1}([a, b])$, then

$$
\int_{a}^{b} f^{\prime}(t) d t=f(b)-f(a)
$$

and $f$ is absolutely continuous.

Proof. We know from Theorem 1 that, for all $\varepsilon>0$, there exists an upper hemicontinuous function $v$ such that $f^{\prime} \leq v$. Choosing a constant $c>0$ in a suitable way, there exists a lower hemicontinous function $g=v+c$ such that $f^{\prime}<g$ and $\int_{a}^{b} g(t) d t<\int_{a}^{b} f^{\prime}(t) d t+\varepsilon$. 
For all $\eta>0$ we define

$$
F_{\eta}(x)=\int_{a}^{x} g(t) d t-f(x)+f(a)+\eta(x-a),
$$

with $a \leq x \leq b$.

Let $x \in[a, b)$. Since $f^{\prime}(x)<g(x)$ and $g(\cdot)$ is lower hemicontinuous, then there exists $(x, x+\delta)$ such that $t \in(x, x+\delta)$ implies that $f^{\prime}(x)<g(t)$ and

$$
\frac{f(t)-f(x)}{t-x}<f^{\prime}(x)+\eta,
$$

because $f(\cdot)$ is differentiable at $x$.

In addition, we have

$$
\begin{aligned}
F_{\eta}(t)-F_{\eta}(x) & =\int_{x}^{t} g(s) d s-(f(t)-f(x))+\eta(t-x) \\
& >f^{\prime}(x)(t-x)-\left(f^{\prime}(x)+\eta\right)(t-x)+\eta(t-x) \\
& =0 .
\end{aligned}
$$

Since $F_{\eta}(a)=0$ and $F_{\eta}(\cdot)$ is continuous, we may define $x^{*}=\sup _{x \in[a, b]}$. If $x^{*}<b$, then by the calculation above we have $F_{\eta}(t)>0$ for $t \in(x, b]$. Therefore, $F_{\eta}(b) \geq 0$. Using the definition (4) we have

$$
F_{\eta}(b)=\int_{a}^{b} g(t) d t-f(b)+f(a)+\eta(b-a) \geq 0
$$

and as $\eta \rightarrow 0^{+}$

$$
\begin{aligned}
\int_{a}^{b} g(t) d t & \geq f(b)-f(a) \\
\int_{a}^{b} f^{\prime}(t) d t+\varepsilon & >f(b)-f(a),
\end{aligned}
$$

where we use (5). As $\varepsilon \rightarrow 0^{+}$,

$$
\int_{a}^{b} f^{\prime}(t) d t \geq f(b)-f(a) .
$$


In repeating the process to $-f^{\prime}$, we obtain

$$
\begin{gathered}
\int_{a}^{b}-f^{\prime}(t) d t \geq(-f(b))-(-f(a)) \\
\int_{a}^{b} f^{\prime}(t) d t \leq f(b)-f(a) .
\end{gathered}
$$

Moreover, by the Lebesgue Theorem, if $f^{\prime}$ exists almost everywhere and it is integrable and $\int_{a}^{b} f^{\prime}(t) d t=f(b)-f(a)$ holds, then $f$ is absolutely continuous.

Observe that whole demonstration was made for unidimensional case, but the generalization is direct if we require existence of $\frac{\partial f}{\partial t_{i}}$ in all utility function's domain (in this case the type spaces becomes $I=\times_{i=1}^{k}[a, b]$ ) and that $\frac{\partial f}{\partial t_{i}} \in L^{1}([a, b])$ for every $i=1, \ldots, k$. For example, with these assumptions theorem 4 of [1] would be obtained by the same procedure and we would have the integral representation to multidimensional case directly. Also, notice that differentiability is the only one additional requirement in the theorem. In fact, in requiring that $f^{\prime} \in L^{1}([a, b])$ we do not impose any other additional assumption, since it is equivalent to the integrable function $b(\cdot)$ proposed by [8] in their Theorem 3.

\section{Concluding Remarks}

Since [9] the importance of representing the value function in mechanism design models as an integral has been highlighted. Several applications have used this result in order to simplify the solving process. Examples are the seminal papers of [10], [2] and [7]. However, all this literature assumes differentiable mechanisms. As the applications have been developed, examples of discontinuous mechanims have raised and that standard framework has became unsatisfactory. In recent years, studies like [8] has overcame this limitation by adding the assumption of absolute continuity of the utility function. Yet, part of literature considers this assumption not elegant and with no economic meaning.

In this paper we propose an alternative assumption for the integral representation of the value function of [8]. Instead of requiring that utility function has derivative almost everywhere, we impose that it has derivative in all its domain. The idea is to obtain conditions in order to apply the Lebesgue Theorem 
which provides at the same time an absolutely continuous value function and its integral representation. Our assumption is technically stronger than that of [8] but we argue that there is a substantial gain of economic interpretation in adding it. While it is difficult to interpret absolute continuity in terms of agent's preferences, the existence of the derivative everywhere means that all agent's choices are smooth.

\section{References}

[1] J. P. Araújo, M. Griebeler, General envelope theorem for multidimensional type spaces, Analls of XXXI Meeting of the Brazilian Econometric Society, 1 (2009).

[2] D. Baron, R. Myerson, Regulating a monopolist with unknown costs, Econometrica, 50 (1982), 911-930, doi: 10.2307/1912769.

[3] K. Chung, W. Olszewski, A non-differentiable approach to revenue equivalence, Theoretical Economics, 2 (2007), 469-487.

[4] J. Ely, Revenue equivalence without differentiability assumptions, Mimeo, Northwestern University (2001).

[5] V. Krishna, E. Maenner, Convex potencials with an application to mechanism design, Econometrica, 69 (2001), 1113-1119, doi: 10.1111/14680262.00233 .

[6] J. Laffont, J. Tirole, A theory of incentives in procurement and regulation, MIT Press, Cambridge (1993).

[7] E. Maskin, J. Riley, Monopoly with incomplete information, RAND Journal of Economics, 15 (1984), 171-196, doi: 10.2307/2555674.

[8] P. Milgrom, I. Segal, Envelope theorems for arbitrary choice sets, Econometrica, 70 (2002), 583-601, doi: 10.1111/1468-0262.00296.

[9] J. Mirrlees, An exploration in the theory of optimum income taxation, Review of Economic Studies 38 (1971), 175-208, doi: 10.2307/2296779

[10] M. Mussa, S. Rosen, Monopoly and product quality, Journal of Economic Theory, 18 (1978), 301-317, doi: 10.1016/0022-0531(78)90085-6.

[11] R. Myerson, Game theory: analysis of conflicts, Harvard Press, Cambridge (1991). 
[12] H. Royden, Real analysis, second edition, The Macmillan Company, New York (1968).

[13] W. Rudin, Real and complex analysis, third edition, McGraw-Hill Science, New York (1986).

[14] S. Willians, A characterization of efficient, bayesian incentive compatible mechanism, Economic Theory, 14 (1999), 155-180, doi: $10.1007 / \mathrm{s} 001990050286$. 
